\title{
Investigation by Raman Spectroscopy of the Decomposition Process of HKUST-1 upon Exposure to Air
}

\author{
Michela Todaro, ${ }^{1,2}$ Antonino Alessi, ${ }^{1,3}$ Luisa Sciortino, ${ }^{1}$ Simonpietro Agnello, \\ Marco Cannas, ${ }^{1}$ Franco Mario Gelardi, ${ }^{1}$ and Gianpiero Buscarino ${ }^{1}$ \\ ${ }^{1}$ Dipartimento di Fisica e Chimica, Università di Palermo, 90123 Palermo, Italy \\ ${ }^{2}$ Dipartimento di Fisica e Astronomia, Università di Catania, 95123 Catania, Italy \\ ${ }^{3}$ Laboratoire H. Curien, UMR CNRS 5516, Université de Lyon, 42000 Saint-Etienne, France \\ Correspondence should be addressed to Gianpiero Buscarino; gianpiero.buscarino@unipa.it
}

Received 29 August 2016; Revised 28 October 2016; Accepted 14 November 2016

Academic Editor: Artem E. Masunov

Copyright (c) 2016 Michela Todaro et al. This is an open access article distributed under the Creative Commons Attribution License, which permits unrestricted use, distribution, and reproduction in any medium, provided the original work is properly cited.

\begin{abstract}
We report an experimental investigation by Raman spectroscopy of the decomposition process of Metal-Organic Framework (MOF) HKUST-1 upon exposure to air moisture $(T=300 \mathrm{~K}, 70 \%$ relative humidity). The data collected here are compared with the indications obtained from a model of the process of decomposition of this material proposed in literature. In agreement with that model, the reported Raman measurements indicate that for exposure times longer than 20 days relevant irreversible processes take place, which are related to the occurrence of the hydrolysis of $\mathrm{Cu}-\mathrm{O}$ bonds. These processes induce small but detectable variations of the peak positions and intensities of the main Raman bands of the material, which can be related to $\mathrm{Cu}-\mathrm{Cu}, \mathrm{Cu}-\mathrm{O}$, and $\mathrm{O}-\mathrm{C}-\mathrm{O}$ stretching modes. The critical analyses of these changes have permitted us to obtain a more detailed description of the process of decomposition taking place in HKUST-1 upon interaction with moisture. Furthermore, the reported Raman data give further strong support to the recently proposed model of decomposition of HKUST-1, contributing significantly to the development of a complete picture of the properties of this considerable deleterious effect.
\end{abstract}

\section{Introduction}

Metal-Organic Frameworks (MOFs) are a new class of hybrid compounds constituted by combination of metallic clusters and organic linkers [1-4]. The formation of a crystalline porous matrix is achieved by reacting these two basic elements in appropriate synthesis conditions [1-4]. The possibility to adapt crystalline structures to a specific request, in terms of appropriate choice of metallic centers, linkers length, pore dimensions, and high surface area, makes MOFs very interesting in a wide range of application fields as catalysis and biomedical field as drug delivery systems, chemical sensors, gas storage, and so on [1-4].

One of the most promising MOFs from the point of view of fundamental and applied research is HKUST-1, synthesized for the first time in 1999 [5]. It is a reference material for adsorption of small molecules and gases and selective capture and transporting of a large variety of gases. In particular, several recent studies have been focused on the hydrogen, methane, and carbon dioxide storage in order to use alternative clean energy sources and with the intent to eliminate greenhouse gases from the atmosphere [6-9].

The outstanding adsorption properties of HKUST-1 are strictly related to its structural characteristics. Indeed, the cubic crystalline structure of this material, whose chemical composition is $\mathrm{Cu}_{3}(\mathrm{BTC})_{2}\left(\mathrm{H}_{2} \mathrm{O}\right)_{3}$, is composed of two $\mathrm{Cu}^{2+}$ ions linked together by four carboxylate groups to form a paddle-wheel unit. Each carboxylate bridge is part of 1,3,5benzene tricarboxylate (BTC) linker molecule. A picture of this structure is reported in Figure 1.

The crystal structure involves large cavities with diameter of about $9 \AA$ and small pockets with diameter of about $6 \AA$. The principal adsorption sites of polar molecules as $\mathrm{NH}_{3}$ and $\mathrm{H}_{2} \mathrm{O}$ are those located on axial binding site of $\mathrm{Cu}^{2+}$ ion, called open metal sites [5].

The good affinity of HKUST-1 with respect to water molecules has been extensively investigated in the past. Indeed, it is well known that the adsorption of water molecules 


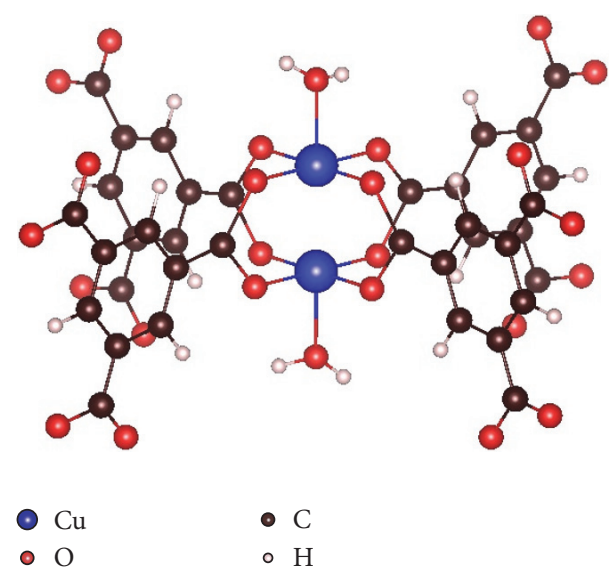

FIGURE 1: Schematic representation of the paddle-wheel unit of as synthesized HKUST-1. Each $\mathrm{Cu}^{2+}$ ion is coordinated with four carboxylate groups, belonging to four 1,3,5-benzene tricarboxylate (BTC) linker molecules and one water molecule.

on open metal sites actually takes place within few minutes of exposure to moisture [10]. This feature has been already taken into account in industrial applications because, for instance, the absorption of some gases is assisted by the presence of water molecules in matrix $[11,12]$.

In spite of this, one of the crucial aspects to consider is the stability of HKUST-1 with respect to a prolonged exposure to moisture, which can affect the crystalline matrix $[13,14]$. Indeed, the range of applications of a MOF is significantly reduced if the integrity of the crystalline matrix is compromised as a consequence of such treatments. In fact, some studies have pointed out that HKUST-1 is not much stable with respect to moisture. In particular, it has been shown that when the material is exposed to moisture, it adsorbs a lot of water molecules until they reach the liquid state into the cavities. When this stage is obtained, water becomes able to hydrolyze the $\mathrm{Cu}-\mathrm{O}$ bonds, breaking the crystalline network of the material. Recently, the effects of the interaction of HKUST-1 with air moisture $(T=300 \mathrm{~K}, 70 \%$ relative humidity) for a long period of time have been investigated in detail by electron paramagnetic resonance (EPR), X-ray diffraction (XRD), scanning electron microscopy (SEM), and surface analysis techniques. It has been shown that the decomposition process of HKUST-1 upon exposure to moisture takes place through three different stages. The first stage, with duration of about 20 days, is characterized by the adsorption of a large amount of water molecules by the material. Water molecules gradually fill the cavities of the material pushing towards the $\mathrm{Cu}^{2+}$ ions. The authors [15] pointed out by XRD that during this first stage the material undergoes a swelling, but no irreversible structural changes take place in the material. The decomposition process of the crystalline matrix actually starts for times longer than 20 days, when the effects of the hydrolysis of the $\mathrm{Cu}-\mathrm{O}$ on the structure of the material become more and more evident on increasing the duration of exposure to air [15]. In particular, during the second stage of the process of decomposition, which takes place for exposure times from 20 days to 50 days, about $35 \%$ of the paddle-wheels become hydrolyzed and consequently one of the four carboxylate bridges involved in these paddle-wheels is detached. Finally, in the third stage of the process of decomposition, which takes place for exposure times longer than 50 days, a second detaching of a carboxylate bridge takes place in the same paddle-wheels already affected in the previous stage of the process.

In order to shed new light on the fundamental issue concerning the structural effects induced by hydrolysis on carboxylate MOFs, here we present an experimental investigation performed by Raman spectroscopy focused on the process of decomposition induced in HKUST-1 upon exposure to air moisture ( $T=300 \mathrm{~K}, 70 \%$ relative humidity) and showing the potentialities of the technique to deepen the understanding of the underneath process in terms of molecular groups changes.

\section{Materials and Methods}

Commercial HKUST-1 in powder form was purchased from Sigma-Aldrich as Basolite C300. A sample of about $10 \mathrm{mg}$ of HKUST-1 was exposed to air at $T=300 \mathrm{~K}$ and relative humidity $(\mathrm{RH})$ of $70 \%$ for different times. We have monitored the vibrational properties of the sample of HKUST-1 with Raman spectroscopy from about 3 hours up to 165 days of exposure to air. Furthermore, we have performed a measurement on the activated sample of HKUST-1.

The Raman measurements were acquired in the region from 150 to $1700 \mathrm{~cm}^{-1}$ with a Bruker Senterra $\mu$-Raman spectrometer equipped with a diode laser working at $\lambda=532 \mathrm{~nm}$. We have performed measurements both at high resolution $\left(3-5 \mathrm{~cm}^{-1}\right)$ and at low resolution $\left(9-15 \mathrm{~cm}^{-1}\right)$. The advantage of the low resolution mode is that it gives spectra with significantly higher signal-to-noise ratio with respect to those obtained in high resolution mode. The nominal laser power was $0.2 \mathrm{~mW}$ to avoid sample modifications. For statistical reasons, each sample was measured in three different points and the resulting spectra were averaged.

The spectrum of the activated sample of HKUST-1 was acquired with a Horiba LabRam HR-Evolution $\mu$-Raman spectrometer with a $532 \mathrm{~nm}$ laser and $1 \mathrm{~mW}$ nominal laser power, at high resolution $\left(4 \mathrm{~cm}^{-1}\right)$. No spectral distortions were induced by laser power.

The sample was put inside a high pressure Linkam THMS600PS cell at $150^{\circ} \mathrm{C}$ (heating ramp $100 \mathrm{C} / \mathrm{min}$ ) in atmospheric pressure and monitored starting from 10 minutes up to 1 hour, recording Raman spectra.

All the recorded spectra were subtracted by a baseline and subsequently normalized with respect to the intensity of the band at $\sim 1000 \mathrm{~cm}^{-1}$. We have chosen this peak for the normalization because it is well known that it is a very stable signal as it is related to the symmetric stretching of the $\mathrm{C}=\mathrm{C}$ bonds in the rigid benzene rings.

\section{Results}

The high resolution Raman spectrum of the sample of activated HKUST-1 and that acquired for the sample after 


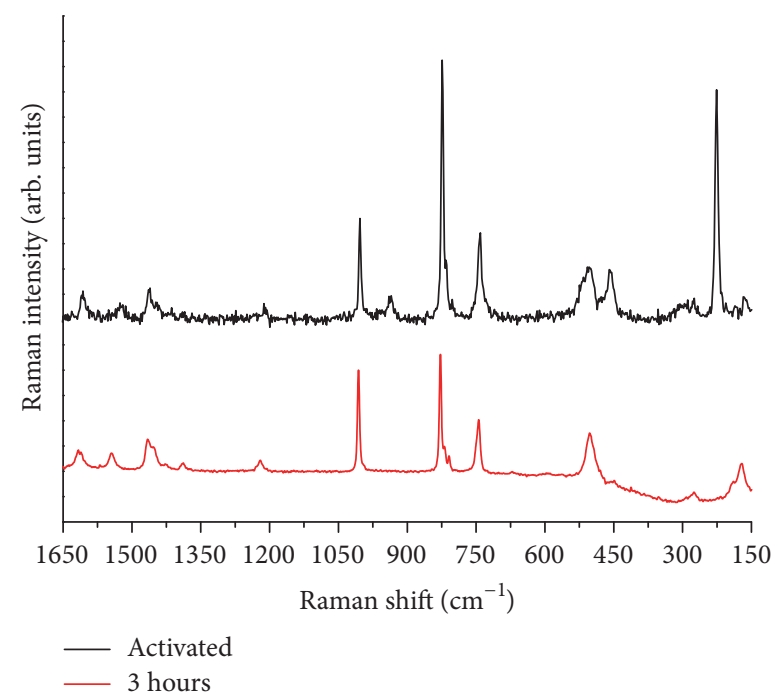

FIgURE 2: High resolution Raman spectra of the HKUST-1 sample activated (black line) and after about 3 hours of exposure to air moisture at $T=300 \mathrm{~K}$ (red line). Spectra are vertically shifted, for viewing purposes, after the normalization described in the text.

about 3 hours of exposure to air $(T=300 \mathrm{~K}, \mathrm{RH} 70 \%)$ are reported in Figure 2.

The two spectra show the same spectroscopic characteristics with respect to those reported in literature for activated and hydrated HKUST-1 $[16,17]$. In particular, the intense peak at about $230 \mathrm{~cm}^{-1}$ evident in the spectrum of activated sample is replaced by two bands in that of the sample exposed to air for 3 hours, in line with results reported by other authors [16].

Regarding the spectrum of the sample exposed to air for 3 hours, between 150 and $600 \mathrm{~cm}^{-1}$, the peaks of vibrational modes involving $\mathrm{Cu}^{2+}$ ions are evident. More precisely, the doublet at $173-191 \mathrm{~cm}^{-1}$ and the peak at $276 \mathrm{~cm}^{-1}$ are assigned in literature to a stretching mode involving $\mathrm{Cu}-\mathrm{Cu}$ dimer and $\mathrm{Cu}-\mathrm{O}_{\mathrm{w}}$, where $\mathrm{O}_{\mathrm{w}}$ indicates the oxygen of the water molecule adsorbed on $\mathrm{Cu}^{2+}$ ion, respectively [16-19]. The doublet at $449-502 \mathrm{~cm}^{-1}$, whose first component is barely detectable, is related to $\mathrm{Cu}-\mathrm{O}$ stretching modes involving oxygen atoms of carboxylate bridges $[16,17]$.

In the central region from 700 to $1100 \mathrm{~cm}^{-1}$, it is possible to recognize the peaks related to the vibrational modes of benzene rings. In particular, at $745 \mathrm{~cm}^{-1}$ and at $828 \mathrm{~cm}^{-1}$, we observe the $\mathrm{C}-\mathrm{H}$ out-of-plane bending modes of rings, whereas at $1006 \mathrm{~cm}^{-1}$ the symmetric stretching mode of $\mathrm{C}=\mathrm{C}$ is well evident in the Raman spectrum [16-19]. In the region from 1400 to $1700 \mathrm{~cm}^{-1}$, two vibrational modes of carboxylate bridges are present. The first feature at $1460 \mathrm{~cm}^{-1}$ corresponds to the symmetric stretching of $\mathrm{O}-\mathrm{C}-\mathrm{O}$, whereas the peak at $1544 \mathrm{~cm}^{-1}$ corresponds to the asymmetric stretching of $\mathrm{O}$ C-O [16]. At $1616 \mathrm{~cm}^{-1}$, the $\mathrm{C}=\mathrm{C}$ symmetric stretching of benzene ring is recognized $[16,17]$.

In Figure 3, a comparison of the Raman spectra obtained for the HKUST-1 exposed to air moisture $(T=300 \mathrm{~K}, \mathrm{RH}$ $70 \%)$ for different times is reported.

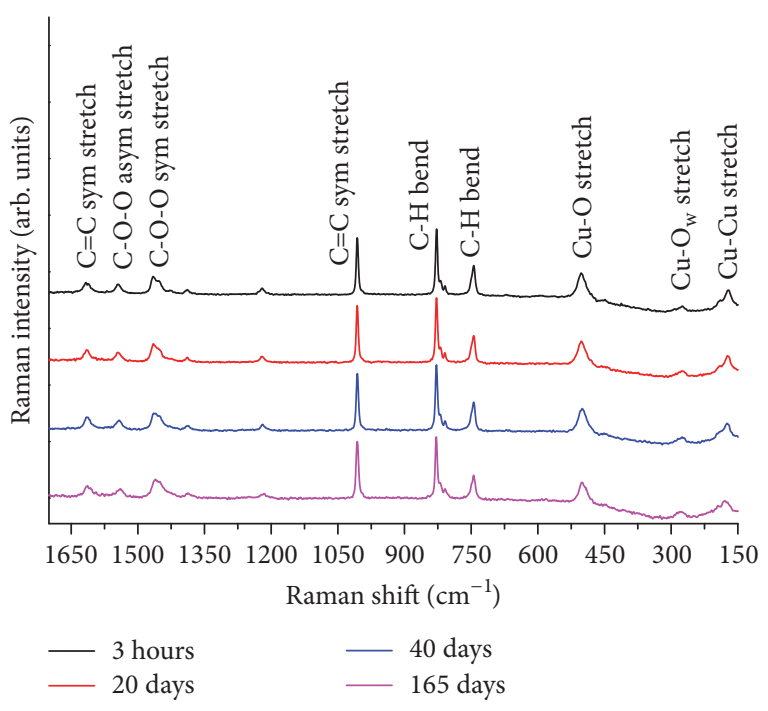

FIGURE 3: High resolution Raman spectra of HKUST-1 acquired after about 3 hours and 20, 40, and 165 days of exposure to air at $T=300 \mathrm{~K}$. Spectra are vertically shifted, for viewing purposes, after the normalization described in the text.

As shown in Figure 3, the Raman spectrum obtained for the sample exposed to air for about 3 hours contains essentially the same peaks as the spectra obtained after longer exposure to air up to 165 days. This indicates that no new vibrations are induced by the decomposition process of the material in the investigated wavenumbers range. In order to obtain a more detailed description of the variations induced in the Raman spectra of HKUST-1 during the exposure of the material to air, we have estimated the intensity and the position of all the relevant peaks present in the Raman spectra. For this analysis we have taken advantages of the high signal-to-noise ratio of the spectra obtained in the low resolution mode.

In Figure 4, the variations induced by exposure of HKUST-1 to air in the intensity and in the position of the peak at about $174 \mathrm{~cm}^{-1}$ attributed to a mode involving the $\mathrm{Cu}-\mathrm{Cu}$ stretching, are presented. A comparison among some indicative spectra in the region of this peak is also reported in Figure 4(b). As shown, we observe that both the amplitude and the peak position of this band remain almost constant up to about 20 days of exposure to moisture. At variance, Figure 4(a) suggests that for longer exposures the peak position undergoes a blue shift and correspondingly the peak amplitude decreases. However, by inspection of the spectra of Figure 4(b), it is easy to recognize that actually the situation is more complex. In fact, it emerges that in correspondence between the longest exposure times the peak exhibits at least two well distinguishable components, both peaked at larger wavenumbers with respect to the position observed after 3 hours of exposure of the material to air moisture.

Similarly, in Figure 5, the changes induced in the peak at about $502 \mathrm{~cm}^{-1}$ related to $\mathrm{Cu}-\mathrm{O}$ stretching are reported. As shown, in this case we have found that this feature remains stable up to about 20 days of exposure to air; thereafter both 


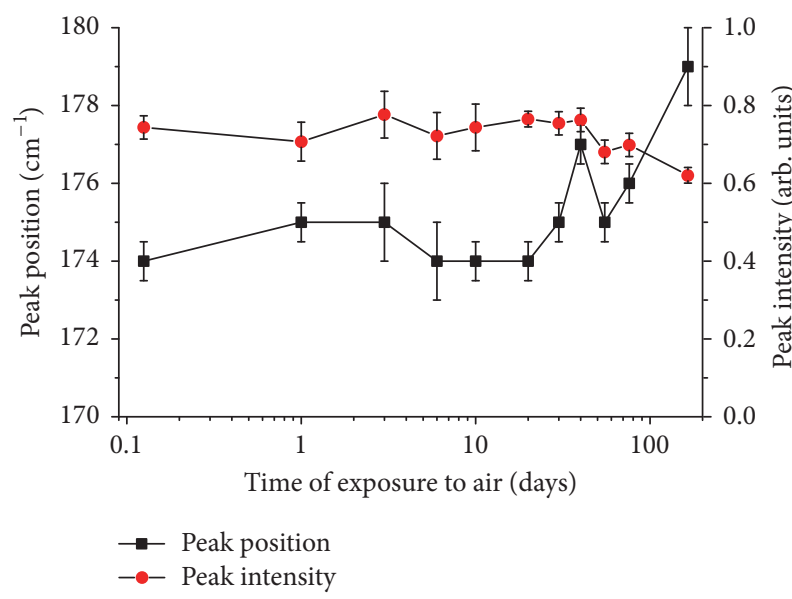

(a)

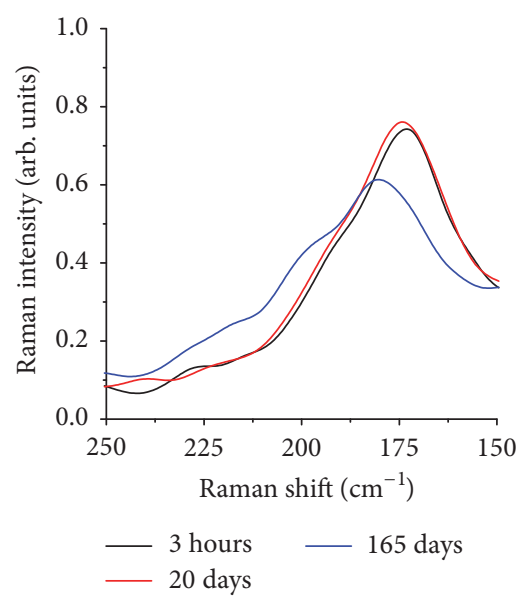

(b)

FIGURE 4: (a) Position (black squares) and intensity (red circles) of the Raman peak at about $174 \mathrm{~cm}^{-1}$ for HKUST-1 as a function of time of exposure to air moisture. (b) Raman spectra in the region of the peak at about $174 \mathrm{~cm}^{-1}$ acquired for HKUST-1 after 3 hours, 20 days, and 165 days of exposure to air moisture.

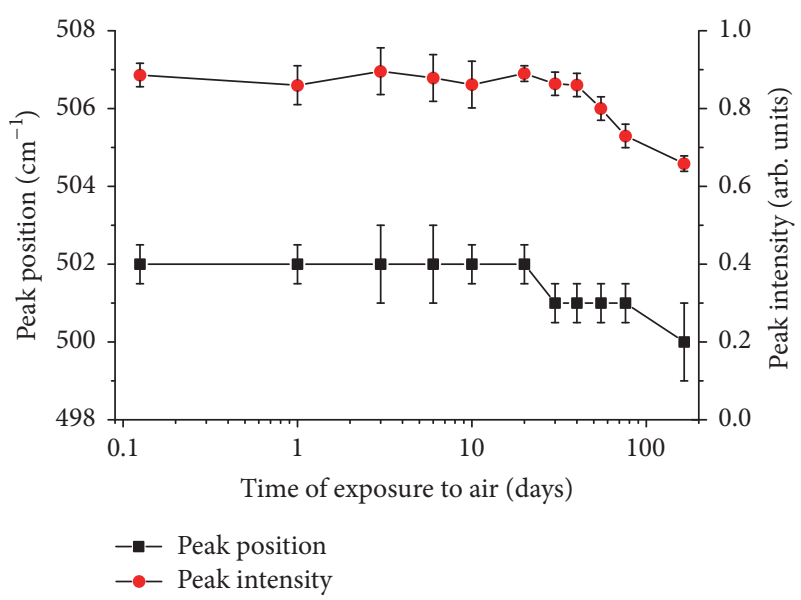

(a)

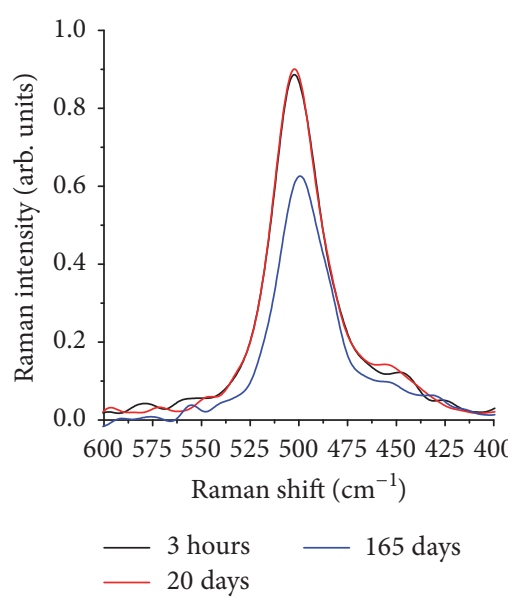

(b)

Figure 5: (a) Position (black squares) and intensity (red circles) of the Raman peak at about $502 \mathrm{~cm}^{-1}$ for HKUST-1 as a function of time of exposure to air moisture. (b) Raman spectra in the region of the peak at about $502 \mathrm{~cm}^{-1}$ acquired for the HKUST-1 after 3 hours, 20 days, and 165 days of exposure to air moisture.

the amplitude and the position of the peak gradually change. The former reduces from 0.88 to 0.64 for exposure to air up to 165 days, whereas the latter undergoes a red shift of about $2 \mathrm{~cm}^{-1}$. This shift is small but well reliable, as it falls above the experimental uncertainty, as it is evident from Figure 5(a). The amplitude difference of the peak at about $502 \mathrm{~cm}^{-1}$ between spectra at 20 and 165 days of exposure to moisture is well recognizable in Figure 5(b).

Finally, in Figure 6, the data concerning the peak at about $1615 \mathrm{~cm}^{-1}$ are summarized. In this case we observe that the peak position remains essentially unchanged during the first 20 days of exposure of the material to air, whereas it undergoes a red shift for longer exposure times. The maximum shift observed for this band is of about $2 \mathrm{~cm}^{-1}$. At variance, no relevant changes of the peak intensity take place during 165 days of exposure of the material to air moisture. Features similar to those here described were also found for the peaks at $1460 \mathrm{~cm}^{-1}$ and at $1544 \mathrm{~cm}^{-1}$ due to the symmetric and asymmetric stretching of O-C-O groups. All the other peaks not mentioned above were found to be essentially not affected by the exposure of the material to air moisture up to 165 days and will not be considered further.

\section{Discussion}

The main purpose of the present investigation is to better understand the processes of decomposition taking place in the carboxylate MOF HKUST-1 upon exposure to air moisture. Since in a recent work [15] a model has been proposed for this process, here we expect to find further support to 


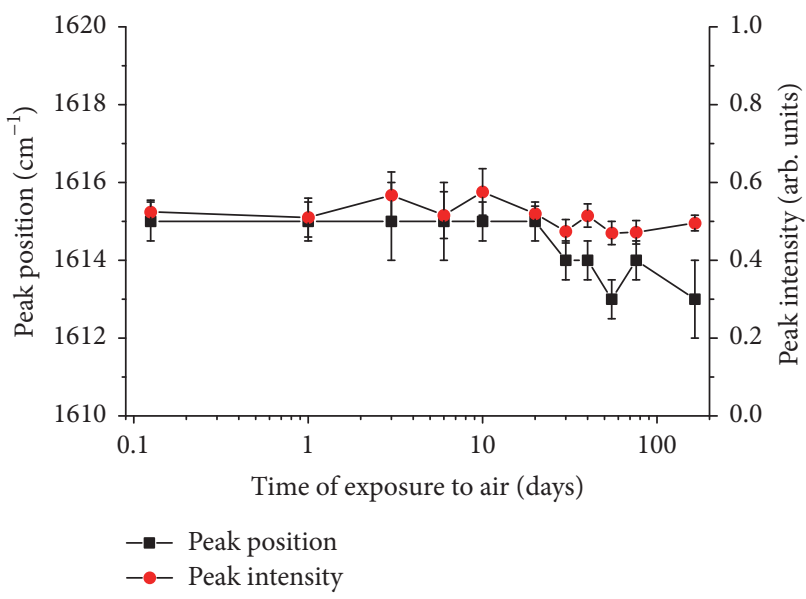

(a)

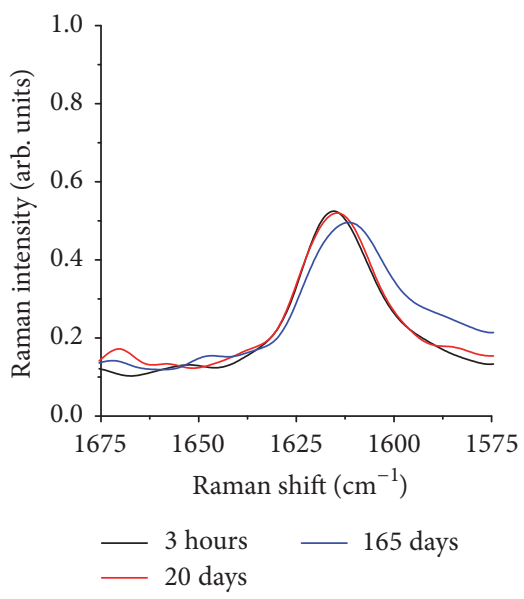

(b)

Figure 6: (a) Position (black squares) and intensity (red circles) of the Raman peak at about $1615 \mathrm{~cm}^{-1}$ for HKUST-1 as a function of time of exposure to air moisture. (b) Raman spectra in the region of the peak at about $1615 \mathrm{~cm}^{-1}$ acquired for HKUST-1 after 3 hours, 20 days, and 165 days of exposure to air moisture.

that model and to obtain a more detailed knowledge of the process. This goal is actually achieved by the overall data presented in the previous section and discussed in detail in the following.

The proposed model indicates that, during the first 20 days of exposure of HKUST-1 to air moisture, no irreversible processes take place. In fact, during this first stage, large quantities of water molecules are absorbed by the material, just filling the volume of the cavities. In agreement with the expectation that no irreversible changes take place during the first 20 days of exposure, the Raman data reported here indicate no relevant changes affecting the peaks falling within the range of investigated wavenumbers. Model [15] also suggests that, during this first stage of the process, the electronic energy levels of the $\mathrm{Cu}^{2+}$ ions significantly change and that the interaction between the two $\mathrm{Cu}^{2+}$ ions within the paddle-wheels becomes stronger, as a consequence of the interaction of these ions with the large quantities of water filling the cavities and pushing in all directions. This effect is actually not caught by the Raman spectroscopy in the present investigation, as no change in the properties of the $\mathrm{Cu}-\mathrm{Cu}$ vibration is recognized. The reason for this failure is probably due to the fact that the two $\mathrm{Cu}^{2+}$ are very far away with respect to each other and consequently their bond strength is expected to be very weak. In fact, the estimated distance between them is of about $2.64 \AA$ [16]. Since the variations of the properties of this bond induced by exposure of the material to air moisture are expected to be small perturbations of the pristine small value, they presumably fall below the detection limit of our Raman equipment.

On the basis of previous reports $[15,18,19]$, it is known that upon exposure of HKUST-1 to air moisture for times between 20 and 40 days the irreversible process of hydrolysis of the $\mathrm{Cu}-\mathrm{O}$ bonds is active. As a consequence of this process one of the four carboxylate bridges is detached in about $35 \%$ of the paddle-wheels of the material, generating a paramagnetic defect named $\mathrm{E}_{1}^{\prime}(\mathrm{Cu})$ center, which involves a $\mathrm{Cu}$ ion in +1 oxidation state with reduced coordination and $\mathrm{a} \mathrm{Cu}^{2+}$ ion with the pristine coordination but modified environment [15]. Finally, the last stage of the process of decomposition of HKUST-1 starts for exposure times longer than 50 days and it involves a further detaching of a second carboxylate bridge in the same paddle-wheels already affected in the previous stage of the process [15]. Such final structure has been attributed to a paramagnetic defect named $\mathrm{E}_{2}^{\prime}(\mathrm{Cu})$ center. The occurrence of relevant irreversible modification of the material for exposure times longer than 20 days is actually confirmed by the reported Raman data. As shown in Figures 4,5 , and 6 , some of the Raman bands suffer detectable peak shifts for such exposure times. More in detail, the blue shift of the peak related to the $\mathrm{Cu}-\mathrm{Cu}$ bonds shown in Figure 4 indicates that the structure of the dimer significantly changes after prolonged exposure to air moisture. In addition, the fact that this peak exhibits two distinguishable components after 165 days of exposure is very interesting, as it indicates that the paddle-wheels of the material are of two different types, with different structures, and characterized by well distinguishable $\mathrm{Cu}-\mathrm{Cu}$ vibrations. The presence of two structures presumably corresponds to the hydrolyzed and nonhydrolyzed paddlewheels, within the pristine structure. On the basis of the model [15], the relative weight of these two components is about $35 \%$ and $65 \%$, respectively. These quantities are roughly consistent with the relative amplitude of the two components shown in Figure 4 in the range from $150 \mathrm{~cm}^{-1}$ to $200 \mathrm{~cm}^{-1}$. By careful inspection of the peak at about $174 \mathrm{~cm}^{-1}$ for the spectra obtained for HKUST-1 exposed to air moisture for about 3 hours and 20 days shown in Figure 4(b), a very small shoulder is recognized at about $178 \mathrm{~cm}^{-1}$. This feature is presumably due to a small fraction of preexisting defective paddle-wheels essentially generated during the synthesis of the material. Concerning the properties of the $\mathrm{Cu}-\mathrm{O}$ bonds, together with a notable red shift, it also suffers a considerable reduction of the peak intensity for exposure times longer than 20 days. This interesting result agrees with the model, 
indicating that after hydrolysis many $\mathrm{Cu}^{+1}$ ions suffer a reduction of their coordination number with respect to the pristine structure.

Finally, we note that since the hydrolysis process involves the oxygen of the carboxylate bridge, one expects also to observe a change of the spectroscopic properties of the peaks at $1460 \mathrm{~cm}^{-1}$ and at $1544 \mathrm{~cm}^{-1}$, corresponding to the symmetric and asymmetric stretching of O-C-O groups. Also this expectation has been fulfilled in our present investigation. As a matter of fact, we have observed a gradual red shift of these bands for long exposure times of the material to air. It is worth noticing that, in agreement with the model of decomposition proposed previously [15], we have observed no change of the intensities of these two bands. This is due to the decomposition process of the material that does not change the number of carboxylate bridges but just the properties of their pristine coordination with the $\mathrm{Cu}^{2+}$ ions. We have found similar changes in the peak at $1615 \mathrm{~cm}^{-1}$ due to the stretching mode of $\mathrm{C}=\mathrm{C}$ bonds of the benzene ring. This is not surprising because such vibrations actually take place in the aromatic ring of the linker and consequently the modifications occurring in the carboxylate bridges are expected to affect indirectly also the $\mathrm{C}=\mathrm{C}$ vibration properties. Also, in this case, no change in the number of the $\mathrm{C}=\mathrm{C}$ bonds is expected. Accordingly, as shown in Figure 6, there is no change of the intensity of the peak at $1615 \mathrm{~cm}^{-1}$ is recognized.

\section{Conclusions}

We report an experimental investigation by Raman spectroscopy of the decomposition process induced by exposure of the MOF HKUST-1 to air moisture. Our data indicate the occurrence of relevant structural processes taking place in the material for exposure times longer than 20 days. In agreement with previous reports [15] these processes are related to hydrolysis which affects a significant fraction of the $\mathrm{Cu}-\mathrm{O}$ bonds of the crystal, irreversibly reducing the crystalline order of HKUST-1. The coexistence of two types of paddlewheels with different structures, corresponding to hydrolyzed and nonhydrolyzed paddle-wheels, predicted by a previous study [15], has been confirmed by detecting a splitting of the Raman peak attributed to the $\mathrm{Cu}-\mathrm{Cu}$ vibration in two well distinguishable components. The predicted change of the coordination number of a fraction of $\mathrm{Cu}$ ions in the material during the decomposition process [15] is also confirmed by the observation of a reduction of the intensity of the Raman peak at $502 \mathrm{~cm}^{-1}$, attributed to $\mathrm{Cu}-\mathrm{O}$ stretching. Summarizing, the overall data reported here support and extend the model of the process of decomposition of HKUST-1 upon exposure to air recently proposed, contributing to reach a complete and reliable description of this technologically relevant process.

\section{Competing Interests}

The authors declare that there are no competing interests regarding the publication of this paper.

\section{Acknowledgments}

The authors thank the people of the LAMP group (http:// www.unipa.it/lamp/) at the Department of Physics and Chemistry of the University of Palermo for useful discussions. The CHAB laboratories at ATeN center (University of Palermo, http://www.chab.center/home/, Med-CHHAB, PONa3_00273) are acknowledged for the LabRam spectrometer and Linkam equipments use.

\section{References}

[1] H. Furukawa, K. E. Cordova, M. O’Keeffe, and O. M. Yaghi, "The chemistry and applications of metal-organic frameworks," Science, vol. 341, no. 6149, Article ID 1230444, pp. 1-12, 2013.

[2] J. L. C. Rowsell and O. M. Yaghi, "Metal-organic frameworks: a new class of porous materials," Microporous and Mesoporous Materials, vol. 73, no. 1-2, pp. 3-14, 2004.

[3] J. Canivet, A. Fateeva, Y. Guo, B. Coasne, and D. Farrusseng, "Water adsorption in MOFs: fundamentals and applications," Chemical Society Reviews, vol. 43, no. 16, pp. 5594-5617, 2014.

[4] L. Sciortino, A. Alessi, F. Messina, G. Buscarino, and F. M. Gelardi, "Structure of the FeBTC metal-organic framework: a model based on the local environment study," The Journal of Physical Chemistry C, vol. 119, no. 14, pp. 7826-7830, 2015.

[5] S. S.-Y. Chui, S. M.-F. Lo, J. P. H. Charmant, A. G. Orpen, and I. D. Williams, "A chemically functionalizable nanoporous material $\left[\mathrm{Cu}_{3}(\mathrm{TMA})_{2}(\mathrm{H} 2 \mathrm{O})_{3}\right]_{n}$, Science, vol. 283, no. 5405, pp. 1148-1150, 1999.

[6] J.-R. Li, R. J. Kuppler, and H.-C. Zhou, "Selective gas adsorption and separation in metal-organic frameworks," Chemical Society Reviews, vol. 38, no. 5, pp. 1477-1504, 2009.

[7] K.-S. Lin, A. K. Adhikari, C.-N. Ku, C.-L. Chiang, and H. Kuo, "Synthesis and characterization of porous HKUST-1 metal organic frameworks for hydrogen storage," International Journal of Hydrogen Energy, vol. 37, no. 18, pp. 13865-13871, 2012.

[8] Y. Liu, C. M. Brown, D. A. Neumann, V. K. Peterson, and C. J. Kepert, "Inelastic neutron scattering of $\mathrm{H}_{2}$ adsorbed in HKUST1," Journal of Alloys and Compounds, vol. 446-447, pp. 385-388, 2007.

[9] J. Getzschmann, I. Senkovska, D. Wallacher et al., "Methane storage mechanism in the metal-organic framework $\mathrm{Cu}_{3}(\mathrm{btc})_{2}$ : an in situ neutron diffraction study, Microporous and Mesoporous Materials, vol. 136, no. 1-3, pp. 50-58, 2010.

[10] F. Gul-E-Noor, D. Michel, H. Krautscheid, J. Haase, and M. Bertmer, "Time dependent water uptake in $\mathrm{Cu}_{3}(\mathrm{btc})_{2}$ MOF: identification of different water adsorption states by ${ }^{1} \mathrm{H}$ MAS NMR," Microporous and Mesoporous Materials, vol. 180, pp. 813, 2013.

[11] N. C. Burtch, H. Jasuja, and K. S. Walton, "Water stability and adsorption in metal-organic frameworks," Chemical Reviews, vol. 114, no. 20, pp. 10575-10612, 2014.

[12] A. Özgür Yazaydin, A. I. Benin, S. A. Faheem et al., "Enhanced $\mathrm{CO}_{2}$ adsorption in metal-organic frameworks via occupation of open-metal sites by coordinated water molecules," Chemistry of Materials, vol. 21, no. 8, pp. 1425-1430, 2009.

[13] J. B. Decoste, G. W. Peterson, B. J. Schindler, K. L. Killops, M. A. Browe, and J. J. Mahle, "The effect of water adsorption on the structure of the carboxylate containing metal-organic frameworks Cu-BTC, Mg-MOF-74, and UiO-66," Journal of Materials Chemistry A, vol. 1, no. 38, pp. 11922-11932, 2013. 
[14] Z. Liang, M. Marshall, and A. L. Chaffee, " $\mathrm{CO}_{2}$ adsorptionbased separation by metal organic framework (Cu-BTC) versus zeolite (13X)," Energy \& Fuels, vol. 23, no. 5, pp. 2785-2789, 2009.

[15] M. Todaro, G. Buscarino, L. Sciortino et al., "Decomposition process of carboxylate MOF HKUST-1 unveiled at the atomic scale level," The Journal of Physical Chemistry C, vol. 120, no. 23, pp. 12879-12889, 2016.

[16] C. Prestipino, L. Regli, J. G. Vitillo et al., "Local structure of framework $\mathrm{Cu}(\mathrm{II})$ in HKUST-1 metallorganic framework: spectroscopic characterization upon activation and interaction with adsorbates," Chemistry of Materials, vol. 18, no. 5, pp. 13371346, 2006.

[17] N. R. Dhumal, M. P. Singh, J. A. Anderson, J. Kiefer, and H. J. Kim, "Molecular interactions of a Cu-based metal-organic framework with a confined imidazolium-based ionic liquid: a combined density functional theory and experimental vibrational spectroscopy study," Journal of Physical Chemistry C, vol. 120, no. 6, pp. 3295-3304, 2016.

[18] K. Tan, N. Nijem, P. Canepa et al., "Stability and hydrolyzation of metal organic frameworks with paddle-wheel SBUs upon hydration," Chemistry of Materials, vol. 24, no. 16, pp. 3153-3167, 2012.

[19] K. Tan, N. Nijem, Y. Gao et al., "Water interactions in metal organic frameworks," CrystEngComm, vol. 17, no. 2, pp. 247-260, 2015. 

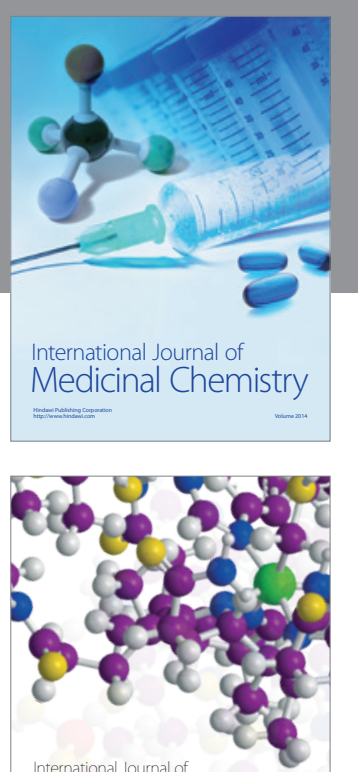

Carbohydrate Chemistry

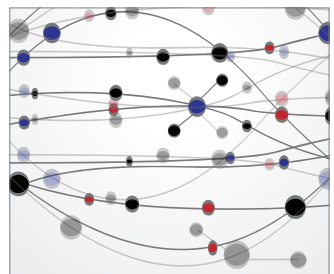

The Scientific World Journal
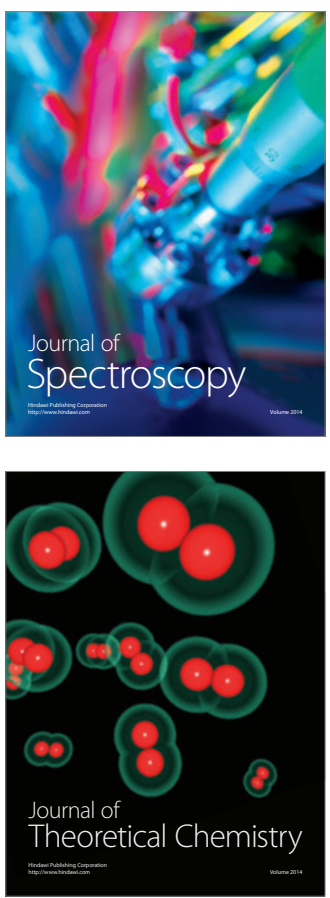
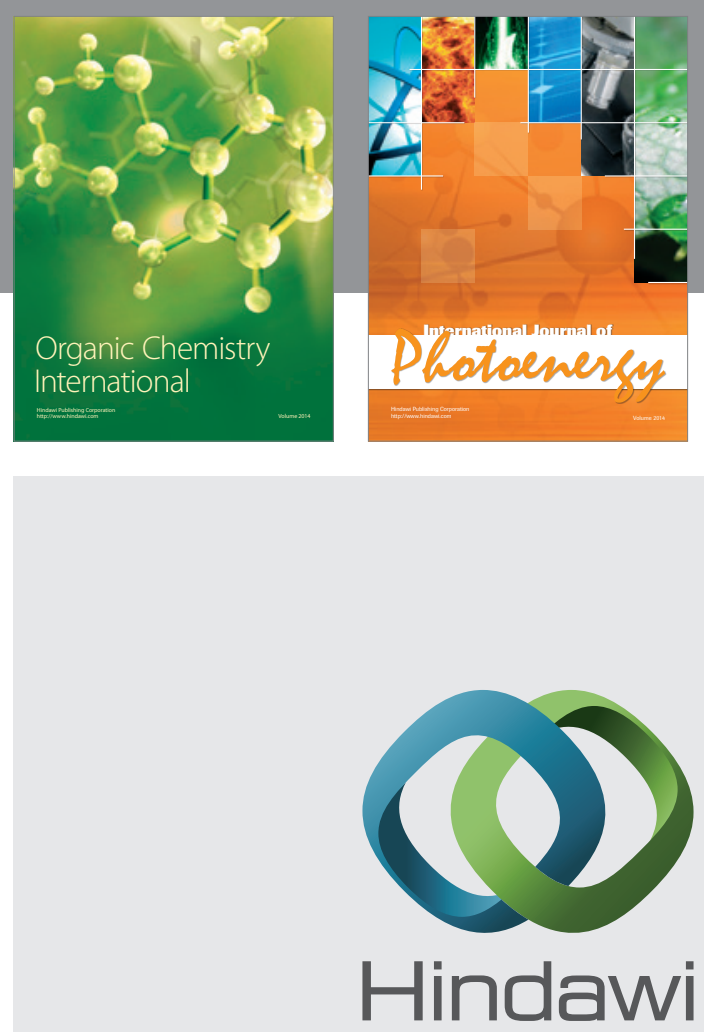

Submit your manuscripts at

http://www.hindawi.com

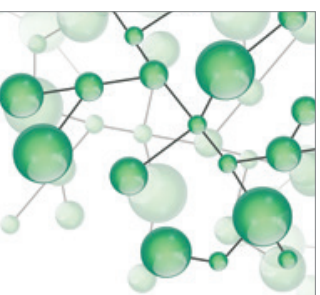

International Journal of

Inorganic Chemistry

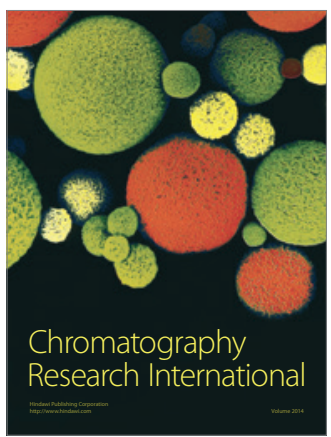

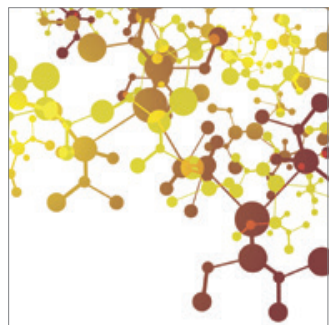

Applied Chemistry
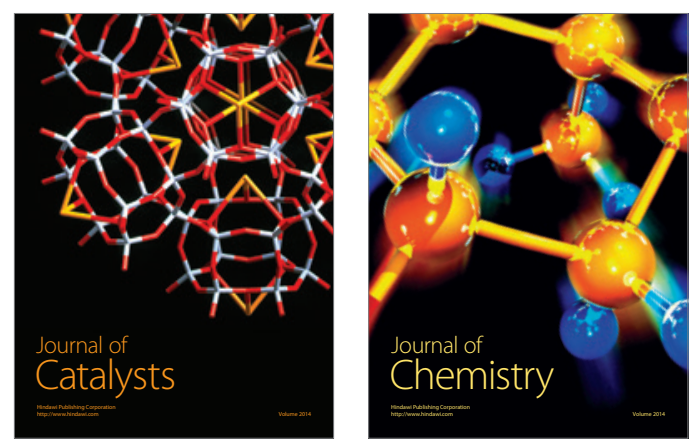
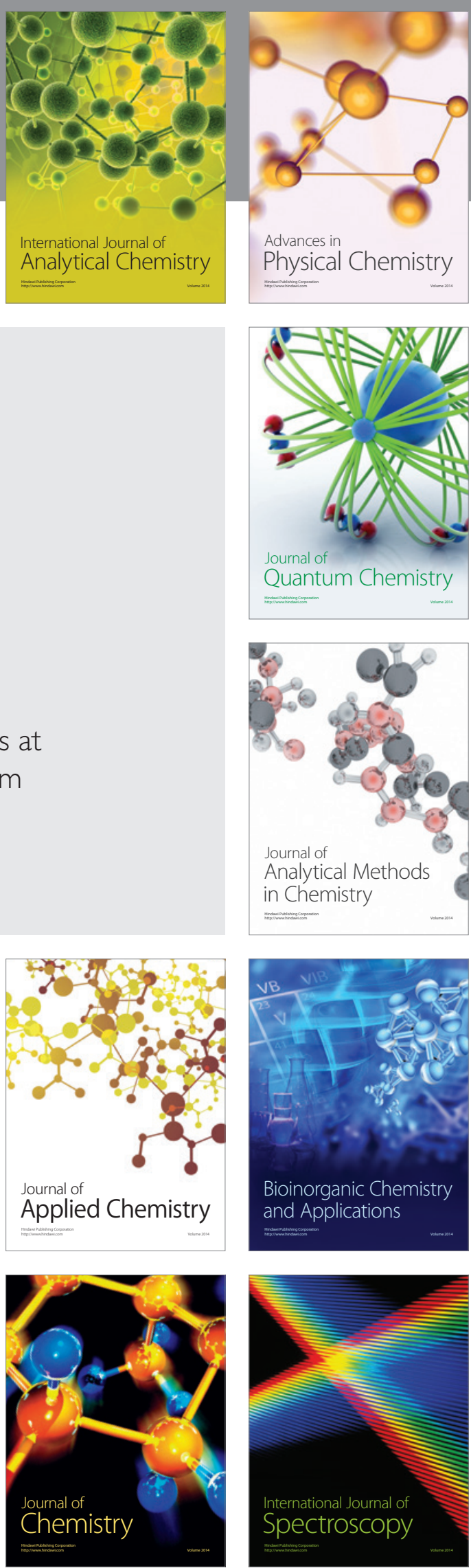